\title{
Population growth study of the rotifer Brachionus sp. fed with triazine- exposed microalgae
}

\author{
C. Rioboo, R. Prado, C. Herrero, A. Cid1
}

Aquatic Toxicology, Volume 83, Issue 4, 1 August 2007, Pages 247-253

Received 23 January 2007, Revised 19 April 2007, Accepted 19 April 2007, Available online 27 April 2007

DOI: 10.1016/j.aquatox.2007.04.006

\begin{abstract}
Few data exist on potential toxic effects that pollutants may have on zooplankton fed microalgae exposed to pesticides. For that reason, microalgal cultures were exposed to different concentrations of the triazine herbicide terbutryn, and used as exclusive food source to Brachionus sp. females, with the aim to evaluate potential deleterious effects upon population growth, survival, reproduction and feeding of the rotifer.Chlorella vulgaris cells were able to accumulate terbutryn, removing more than $90 \%$ of the total amount of herbicide in all the exposed cultures. Growth curves of Brachionus sp. showed that population density decreased as terbutryn concentration increased in the microalgal cells. In fact, this species of rotifer did not survive beyond four days when fed with microalgae exposed to $500 \mathrm{nM}$ terbutryn. Percentage of reproductive females in rotifer populations fed with terbutryn-exposed microalgae decreased significantly as herbicide concentration increased. In control cultures, reproductive females laid a maximum of three eggs per individual; whereas in $100 \mathrm{nM}$ cultures, reproductive females laid only one egg per individual throughout the treatment period. Terbutryn accumulated in $C$. vulgaris provoked a decrease in the feeding rate ofBrachionus sp. cultures fed with these microalgae with respect to control cultures. After this treatment period, all the rotifer populations, except those fed with $500 \mathrm{nM}$ terbutryn-exposed microalgae, showed recovery patterns when they were returned to fresh medium containing herbicide-free microalga. Taking into account the results obtained, uptake of pesticides by phytoplankton can directly affect higher trophic levels.
\end{abstract}

\section{Keywords}

Microalga; Rotifer; Herbicide; Bioaccumulation; Pesticide transfer

\section{Introduction}

The extensive use of herbicides in agriculture and their persistence in the environment result in many of these compounds being present in surface and ground waters, and must be considered

\footnotetext{
${ }^{1}$ Laboratorio de Microbioloxía, Facultad de Ciencias, Universidade da Coruña, Rúa Alejandro de la Sota $N^{\circ} 1,15008$ A Coruña, Spain
} 
a potential risk for aquatic life as well as for the quality of drinking water (Katsumata et al., 2006). Terbutryn, a s-triazine herbicide, is widely used as control agent for most grasses and many annual broadleaf weeds in cereal and legume fields and under fruit trees (Moretti et al., 2002), and also as an aquatic herbicide to control of submerged and free-floating weeds and algae in waterways, reservoirs and fish ponds (Tomlin, 2003), where it affects many non-target organisms (Arufe et al., 2004).

Compounds with high lipophilicity are biologically available especially for benthic organisms (Gross-Sorokin et al., 2003). Terbutryn is relatively insoluble in water and has the potential to bioaccumulate in organisms.

Chlorella vulgaris is a freshwater microalga, commonly used in toxicity tests and as food in aquaculture. Rotifers are one of the common components of freshwater and play an important role in freshwater ecosystems (Preston and Snell, 2001a). Rotifers are considered to be sensitive indicators of the health of their environment due to their biological characteristics and their role as a food link between phytoplankton and fish ( Snell and Janssen, 1995 and Marcial et al., 2005 and Taylor et al., 2005). In fact, there has been an increasing trend in the use of rotifers as bioassay organisms for ecotoxicological studies in recent years (Sarma et al., 2001a and Sarma et al., 2001b). The model food chain selected in this study consisted of $C$. vulgaris, as primary producer and Brachionussp., as primary consumer. Microalgal cultures exposed during $24 \mathrm{~h}$ to different concentrations of the triazine herbicide terbutryn, were used as exclusive food source to Brachionus sp. females, with the aim to evaluate potential deleterious effects on population growth, survival, reproduction and feeding of the rotifer. A potential recovery of Brachionus sp. populations was also assayed.

\section{Materials and methods}

\subsection{Microalgal assays}

The microalgal species $C$. vulgaris Beijerinck (Oocystaceae) (strain CCAP 211/11B) was obtained from the Culture Collection of Algae and Protozoa (CCAP; Cumbria, UK). For routine cultures and for the experiments, the microalga was cultured in sterilized and filtered seawater, supplemented with Bristol medium (Brown et al., 1967) without sodium chloride. Salinity was adjusted at 10 psu adding sterilized and filtered distilled water. The microalgal inoculum was maintained under semi-continuous conditions, with the aim to use cells growing in a logarithmic phase for all experiments. Cultures were illuminated with light $\left(70 \pm 2 \mu \mathrm{mol}\right.$ photon $\left.\mathrm{m}^{-2} \mathrm{~s}^{-1}\right)$, bubbled with air at a rate of $10 \mathrm{I} \mathrm{min}^{-1}$ and maintained at $18 \pm 1^{\circ} \mathrm{C}$, with a dark:light cycle of 12:12 h.

At time zero, terbutryn was added to cultures at three final concentrations: 12.5, 100 and $500 \mathrm{nM}$. Cultures without herbicide were included as controls. Terbutryn was Riedel de Haën Pestanal standard for environmental analysis. Herbicide stock solutions were prepared by dissolving herbicide in $100 \%$ methanol and filtered through $0.2 \mu \mathrm{m}$ filter membranes. Final 
methanol concentration never exceeded $0.05 \%(\mathrm{v} / \mathrm{v})$ of the final volume of culture; cultures with this maximum volume of methanol but without pesticide were also carried out, showing nondifferences to control cultures without pesticide or methanol.

All cultures were carried out in sterilized glass bottles. Initial density of the cultures was $9 \times 10^{6}$ cells $\mathrm{ml}^{-1}$. After $24 \mathrm{~h}$ of terbutryn exposure, microalgal cells were collected and resuspended in seawater at 10 psu salinity to remove the unabsorbed herbicide of the microalgal cultures.

Microalgal cell density was measured by counting culture aliquots in a Neubauer haemocytometer using an Eclipse E400 microscope (Nikon, Japan).

Fluorescence of cells stained with propidium iodide was analysed to study cell viability in a flow cytometer equipped with an argon laser. Propidium iodide is unable to pass through intact cell membranes; however, when a cell dies the integrity of the cell membrane fails and PI is able to enter and stain the nucleic acids (Ormerod, 1990) and produce red fluorescence when excited with blue light. Hence, PI can be used to discriminate between live non-fluorescent cells and non-viable fluorescent cells. Aliquots containing $1 \times 10^{6} \mathrm{cells} \mathrm{ml}^{-1}$ were stained with $\mathrm{PI}$ to a final concentration of $3 \mu \mathrm{g} \mathrm{ml}^{-1}$ for $15 \mathrm{~min}$.

Terbutryn accumulated in microalgal cells was determined by gas chromatography-mass spectrometry (GC-MS) as described in González-Barreiro et al. (2006).

\subsection{Rotifer assays}

The herbivorous rotifer Brachionus sp. (Brachionidae) was obtained from Veracruz Aquarium, Mexico. Rotifer cultures were carried out using seawater at 10 psu salinity using $C$. vulgaris as exclusive food ( $3 \times 10^{5}$ microalgal cells per rotifer). Rotifers were transferred every four days to fresh medium and fed with the green alga at the same density to maintain the cultures in the logarithmic phase of the amictic growth. For maintenance of cultures as well as for experiments, rotifer cultures were carried out in a thermoregulated chamber at $28 \pm 1^{\circ} \mathrm{C}$, under defined illumination (10 $\pm 1 \mu \mathrm{mol}$ photon $\left.\mathrm{m}^{-2} \mathrm{~s}^{-1}\right)$ with a photoperiod of 12:12 light:dark and cultures were conducted at a pH from 7 to 8 with an aeration flow of $51 \mathrm{~min}^{-1}$.

For the experiments, a static-daily renewal system (batch culture), under the conditions described above, was used. Each experimental culture of Brachionus sp. was carried out in Pyrex glass bottle of $1000 \mathrm{ml}$ capacity containing $400 \mathrm{ml}$ of medium using asexual females of mixed age, at an initial density of five individuals per $\mathrm{ml}$. The $24 \mathrm{~h}$ cultures of the unicellular microalga $C$. vulgaris pre-exposed to different concentrations of the triazine herbicide terbutryn $(12.5,100$ and $500 \mathrm{nM})$, were used as exclusive food source to rotifer females for five days. Rotifers fed with non-treated $C$. vulgaris were used as control. Since terbutryn was prepared in methanol, cultures of Brachionus sp. were fed with microalgae exposed to the maximum volume of methanol and used as a complementary control. No differences were observed between both controls. 
After inoculation, $1 \mathrm{ml}$ rotifer culture aliquots were analysed daily under a stereomicroscope to determine alive and dead females. In addition, percentage of egg-bearing females and the number of eggs per egg-bearing female in cultures were counted in 25 individuals per replicate. After counting the total number of rotifers from each experimental flask, individuals were returned to cultures and algal densities of rotifer cultures were determined to calculate the ingestion rates, expressed as food uptake per rotifer during time $t$. Then, test rotifers were filtered through a $50 \mu \mathrm{m}$ mesh and transferred to fresh medium and terbutryn-treated $C$. vulgaris was added to adjust the cell density to $3 \times 10^{5}$ cell rotifer-1. $^{-1}$.

From the data obtained, rate of population increase (r), expressed in days ${ }^{-1}$, was calculated using the following equation:

$$
r=\frac{(\ln N t-\ln N O)}{t}, \quad \text { where } N O \text { and } N t \text { are the initial and final rotifer population densities }
$$

respectively and $t$ is time in days.

Rotifer generation times (g.t.) in days were calculated from:

$$
\text { g.t. }=t . \ln \left(\frac{N t}{N O}\right) \text {, }
$$

where $t$ is culture days and $N O$ and $N t$ are the initial and final rotifer densities, respectively ( Krebs, 1985).

After the 5th day of experiment (treatment period), all Brachionus sp. populations were introduced to fresh medium and fed with herbicide-free microalga for two days to study the phenomenon of recovery under favourable conditions (recovery period). The experiments were completed after seven days (experiment period).

\subsection{Data analysis}

All experiments were carried out in triplicate with three replicates per terbutryn concentration and data were statistically analysed by a one-way analysis of variance (ANOVA) and, when differences observed were significant, means were compared by the multiple range Duncan test.

\section{Results}

Accumulated terbutryn increased significantly $(F=127334.13$; d.f. $=3,8 ; P<0.001)$ in the microalgal cells as the concentration of the herbicide increased in the medium for all concentrations assayed ( Table 1). The maximum value of herbicide bioaccumulation was 12.15 ng cell-1, reached in cultures of $500 \mathrm{nM}$ ( Table 1).

Table 1.

Bioaccumulation of terbutryn and its effect on the viability of $C$. vulgaris cells after $24 \mathrm{~h}$ of herbicide exposure

Terbutryn concentration (nM) control Bioaccumulation

Viability 


\begin{tabular}{ll|l|l} 
& $\left.(\text { ng terbutryn cell })^{-1}\right)$ & $(\%)$ & $(\%)$ \\
& $0.00 \pm 0.00$ & $0.00 \pm 0.00$ & $99.99 \pm 0.01$ \\
\hline 12.5 & $0.41 \pm 0.01^{\mathrm{a}}$ & $91.61 \pm 0.99^{\mathrm{a}}$ & $99.98 \pm 0.02$ \\
\hline 100 & $3.56 \pm 0.10^{\mathrm{a}}$ & $93.56 \pm 1.60^{-\mathrm{a}}$ & $99.94 \pm 0.01$ \\
\hline 500 & $12.15 \pm 0.61^{\mathrm{a}}$ & $93.77 \pm 1.87^{\mathrm{a}}$ & $99.93 \pm 0.03$
\end{tabular}

At time zero, the culture medium was supplemented with terbutryn at the final concentrations indicated.

Data are given as mean values \pm standard errors of the means.

a Significant differences at a level of significance of $0.05(P<0.05)$

However, no significant differences $(F=3.27$; d.f. $=2,6 ; P>0.05)$ were observed when data are expressed as a percentage of the added herbicide. Terbutryn removed from medium was always higher than $90 \%$ for all concentrations assayed ( Table 1), ranging from $92 \%$ in $12.5 \mathrm{nM}$ cultures to $94 \%$ in the $500 \mathrm{nM}$ cultures.

Cell viability in $C$. vulgaris cultures assayed by FCM was not significantly affected $(F=1.70$; d.f. $=3,8 ; P>0.05$ ) by the addition of terbutryn ( Table 1 ), remaining close to $100 \%$ for all terbutryn concentrations assayed.

Significant growth of Chlorella cells in the rotifer cultures was not observed. Brachionus $s p$. cultures fed with $C$. vulgaris exposed to terbutryn showed lower feeding rates (microalgal cell rotifer-1 $^{-1} \mathrm{~h}^{-1}$ ) than control cultures fed with terbutryn-free microalgal cells ( Fig. 1). During the first day, there were no differences among the feeding rate of any rotifer culture (near

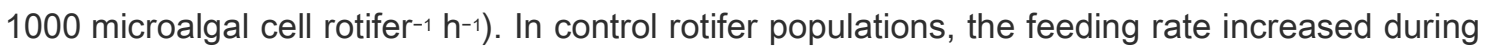
the first four days of experiment, reaching maximum values of 3000 microalgal cell rotifer-1 $\mathrm{h}^{-1}$. Rotifers fed with $100 \mathrm{nM}$ terbutryn-exposed microalgae maintained a constant feeding rate (1000 microalgal cell rotifer-1 $\mathrm{h}^{-1}$ ) from Day 1 to Day 5 and then this rate increased significantly $(F=973.72 ;$ d.f. $=2,6 ; P<0.001)$ during the recovery period, reaching similar values to the maximum observed in control cultures ( Fig. 1). In rotifer cultures fed with the highest triazineexposed microalgae, the feeding rates decreased to 0 at Day 4 and remaining constant through to the end of the experiment period.

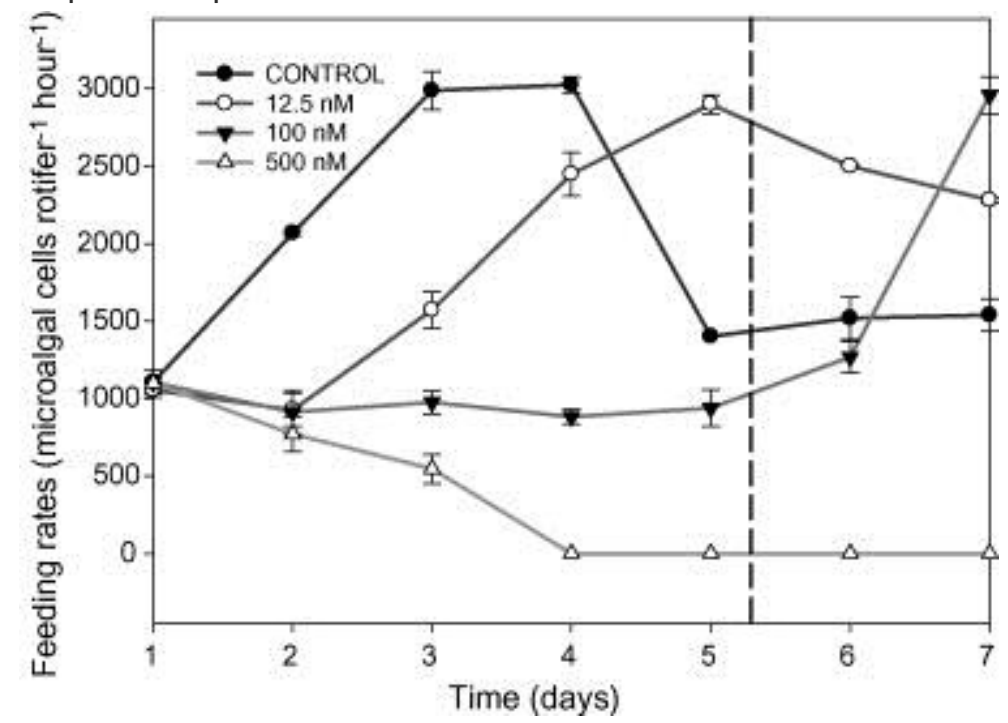

Fig. 1. 
Feeding rates of Brachionus sp. fed with microalgae exposed to different concentrations of terbutryn. A dashed line separates the treatment period from the recovery period. Data are given as mean values \pm standard errors of the means.

Growth curves of Brachionus sp. showed that the population density decreased when compared to control cultures as terbutryn concentration increased in the microalgal cells ( Fig. 2).When rotifers were fed with non-treated $C$. vulgaris (control cultures), the population dynamics showed a typical sigmoid curve, reaching the maximum peak population density recorded in this study after five days of experiment (926.14 individuals $\mathrm{ml}^{-1}$ ), starting a stationary phase after this point ( Fig. 2).

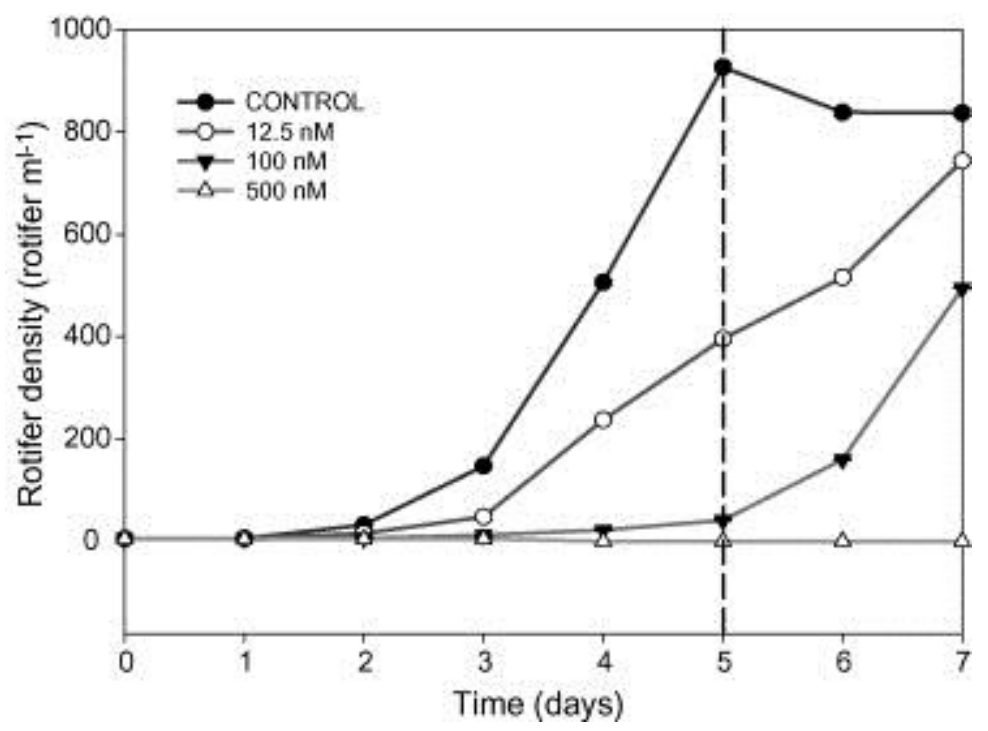

Fig. 2.

Population growth curves of Brachionus sp. fed with microalgae exposed to different concentrations of terbutryn. A dashed line separates the treatment period from the recovery period. Data are given as mean values \pm standard errors of the means.

Rotifer populations fed with terbutryn-exposed Chlorella showed a longer lag phase than control cultures( Fig. 2). Rotifer cultures fed with Chlorella exposed to the lowest assayed concentration $(12.5 \mathrm{nM})$ did not reach the stationary phase at any moment of the assay ( Fig. 2).During the recovery period, these rotifer cultures (exposed to the minimum terbutryn concentration in Chlorella cells) showed the maximum population generation time (3.17 d) of the present study. The density of rotifers fed with $100 \mathrm{nM}$ terbutryn-exposed microalgae increased significantly $(F=3847.98$; d.f. $=2,6 ; P<0.001)$ when they were fed again with herbicide-free microalga during the recovery period, reaching growth rates close to 1 day-1 $^{-1}$ during this period, with the minimum population generation time recorded $(0.88 \mathrm{~d}$ ) ( Table 2). Finally, when rotifers were fed with Chlorellacultures exposed to the maximum terbutryn concentration assayed (500 nM), no growth was observed during the entire experiment period (seven days), including the treatment and the recovery periods ( Table 2, Fig. 2).

Table 2.

Population growth rates and generation times of Brachionus sp. fed with terbutryn-exposed microalgae to different concentrations calculated after the treatment period (0-5 d), for the recovery period (5-7 d) and for the total experimental period $(0-7 \mathrm{~d})$ 


\begin{tabular}{|c|c|c|c|c|c|c|}
\hline \multirow{2}{*}{$\begin{array}{l}\text { Terbutryn } \\
\text { concentration } \\
(\mathrm{nM})\end{array}$} & \multicolumn{2}{|c|}{ Treatment period } & \multicolumn{2}{|c|}{ Recovery period } & \multicolumn{2}{|c|}{ Experimental period } \\
\hline & $\begin{array}{l}\text { Population } \\
\text { growth rate } \\
\left(\mathrm{d}^{-1}\right)\end{array}$ & $\begin{array}{l}\text { Population } \\
\text { generation } \\
\text { time (d) }\end{array}$ & $\begin{array}{l}\text { Population } \\
\text { growth rate } \\
\left(\mathrm{d}^{-1}\right)\end{array}$ & $\begin{array}{l}\text { Population } \\
\text { generation } \\
\text { time (d) }\end{array}$ & $\begin{array}{l}\text { Population } \\
\text { growth rate } \\
\left(\mathrm{d}^{-1}\right)\end{array}$ & $\begin{array}{l}\text { Population } \\
\text { generation } \\
\text { time (d) }\end{array}$ \\
\hline Control & $1.04 \pm 0.01$ & $0.96 \pm 0.01$ & $0.00 \pm 0.00$ & - & $0.73 \pm 0.00$ & - \\
\hline 12.5 & $0.87 \pm 0.00^{\mathrm{a}}$ & $1.14 \pm 0.04^{\mathrm{a}}$ & $0.32 \pm 0.00^{\mathrm{a}}$ & $3.17 \pm 0.11^{\mathrm{a}}$ & $0.71 \pm 0.00^{\mathrm{a}}$ & $3.17 \pm 0.11^{\mathrm{a}}$ \\
\hline 100 & $0.47 \pm 0.00^{\mathrm{a}}$ & $2.14 \pm 0.09^{\mathrm{a}}$ & $1.13 \pm 0.02^{\mathrm{a}}$ & $0.88 \pm 0.02^{-\mathrm{a}}$ & $0.66 \pm 0.00^{\mathrm{a}}$ & $0.88 \pm 0.02^{\underline{a}}$ \\
\hline 500 & $0.00 \pm 0.00^{\mathrm{a}}$ & - & $0.00 \pm 0.00$ & - & $0.00 \pm 0.00^{\mathrm{a}}$ & - \\
\hline
\end{tabular}

Data are given as mean values \pm standard errors of the means.

a

Significant differences $(P<0.05)$

Table options

In control cultures, dead females did not appear until the 5th day, with survival levels of $96 \%$ at the end of the assay (Fig. 3). The survival curves for all Brachionus sp. cultures fed with terbutryn-exposed microalgae showed a significant decrease $(F=1840.22$; d.f. $=3$, 8; $P<$ 0.001) as compared to control cultures until the 4th day of the treatment period ( Fig. 3). During the recovery period, the survival level increased for rotifer cultures fed with Chlorella exposed to the concentration 12.5 and $100 \mathrm{nM}$. After the 4th day of experiment, survival in the rotifer populations fed with the highest assayed concentration of terbutryn reached values of $0 \%$.

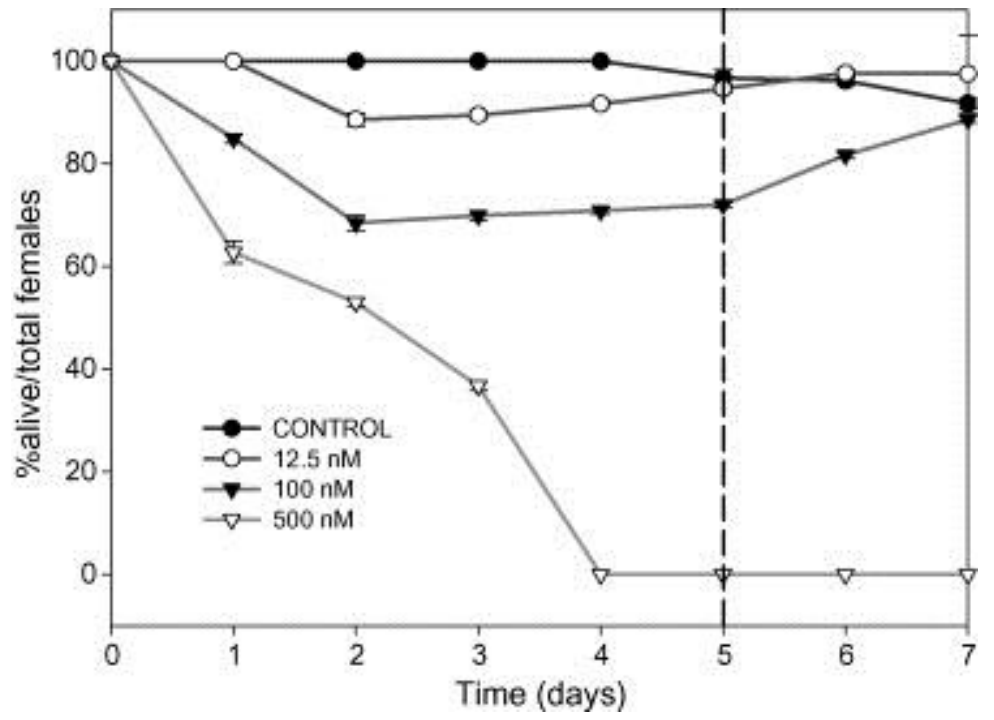

Fig. 3.

Survival curves of Brachionus sp. fed with microalgae exposed to different concentrations of terbutryn.

A dashed line separates the treatment period from the recovery period. Data are given as mean values \pm standard errors of the means.

The reproduction of rotifers was affected by the presence of terbutryn in the microalgal cells used as food. Rotifer cultures fed with terbutryn-exposed $C$. vulgaris showed significant alterations in the percentage of egg-bearing females, expressed as the proportion of the eggbearing females with respect to total number of females ( Fig. 4) and the number of eggs per egg-bearing female ( Fig. 5) as compared to control cultures. In control cultures, the proportion of females with eggs ranged from 90 to $85 \%$ until the 4th day ( Fig. 4) with an egg ratio close to 
three eggs per egg-bearing female at this point ( Fig. 5); and then, the percentage of reproductive females decreased to $30 \%$ at the end of the experimental period, with an egg ratio of one egg per egg-bearing female ( Fig. 4 and Fig. 5). Rotifer populations fed with microalgae exposed to the lowest concentration of triazine maintained a percentage of egg-bearing females around $80 \%$ throughout the experimental period ( Fig. 4). With respect to the number of eggs per egg-bearing female, these cultures showed a similar pattern to that observed in controls during the treatment period, while this number slightly increased during the recovery period ( Fig. 5). The percentage of reproductive females, with an average value of one egg per eggbearing females ( Fig. 5), in rotifer populations fed with microalgae exposed to $100 \mathrm{nM}$ terbutryn decreased significantly $(F=364.82$; d.f. $=3,8 ; P<0.001)$ to $40 \%$ on the 3 rd day. After this point the percentage increased to nearly $90 \%$ at the end of the recovery period (Fig. 4) and the number of eggs per egg-bearing female increased to 2.5 ( Fig. 5). The percentage of reproductive females decreased continuously in the cultures fed with microalgae exposed to the highest herbicide concentration, being $0 \%$ after the 4th day ( Fig. 4); after this point of the experiment, reproductive females were not observed in these cultures ( Fig. 5).

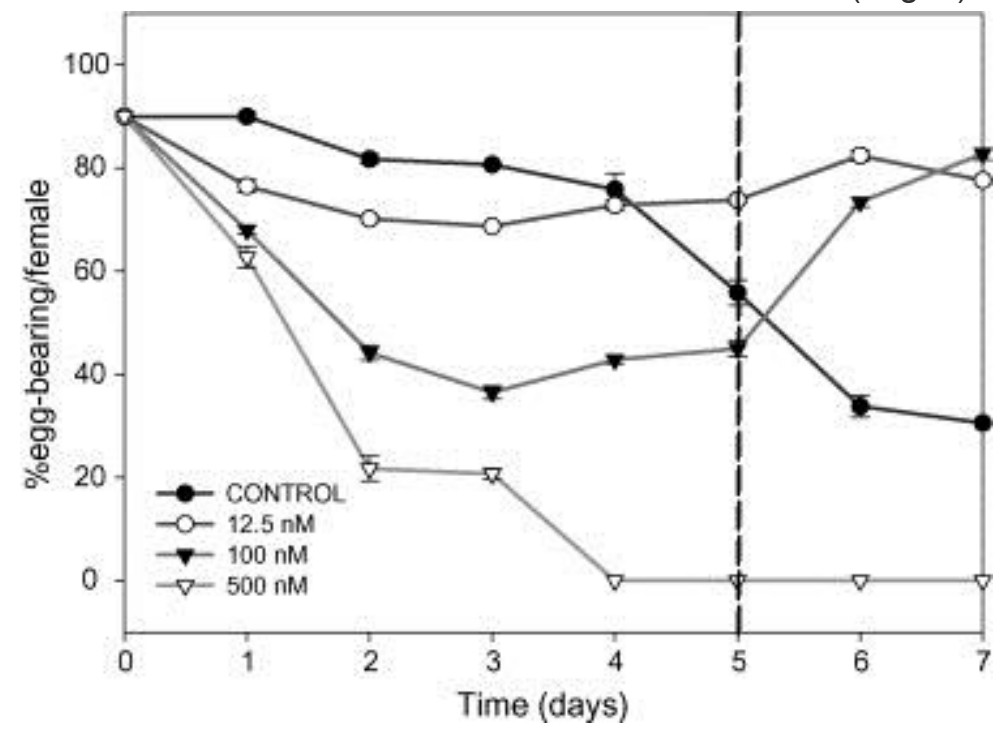

Fig. 4.

Reproduction expressed as percentage of egg-bearing females of Brachionus sp. fed with microalgae exposed to different concentrations of terbutryn. A dashed line separates the treatment period from the recovery period. Data are given as mean values \pm standard errors of the means. 


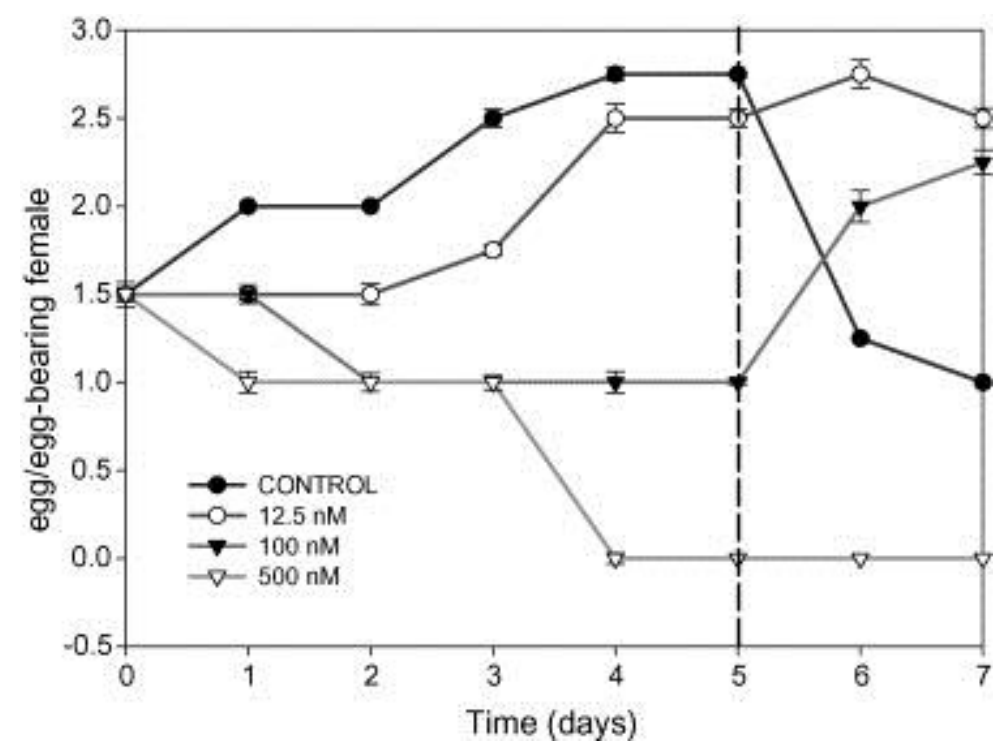

Fig. 5 .

Reproduction expressed as egg ratios of Brachionus sp. cultures fed with microalgae exposed to different concentrations of terbutryn. A dashed line separates the treatment period from the recovery period. Data are given as mean values \pm standard errors of the means.

\section{Discussion}

A key question for the environmental assessment of a chemical is its bioavailability for aquatic life (Richter and Nagel, 2007). Moderately lipophilic compounds, such as terbutryn (log Kow 3.65) (Carabias-Martínez et al., 2003), can be taken up via water (bioconcentration) by unicellular algae and then may be transferred to other aquatic organisms via food (biomagnification). Based on this possibility, the goal of this study was to determine the transfer and the potential toxic effect of this herbicide to primary levels of a food chain.

The results obtained demonstrate that the freshwater microalga $C$. vulgaris showed a high bioconcentration capability for terbutryn, since $24 \mathrm{~h}$ of exposure via water was long enough to allow microalgae to accumulate large amounts of this triazine herbicide. Episodic pollution is not always considered important ( McCahon and Pascoe, 1990), but even short exposure periods of toxicity can affect microalgal populations. Even if low levels of herbicide are detected in water, toxicity can occur due to ingestion of microalgae with bioaccumulated herbicide. Similar results were reported with metals such as copper ( Moreno-Garrido et al., 1999). Although bioconcentration of triazine is a prerequisite for toxicity in microalgal cells ( Tang et al., 1998), terbutryn uptake does not significantly affect the microalgal cell viability after $24 \mathrm{~h}$ of exposure, even at the higher assayed concentrations of the herbicide. Based on this, $C$. vulgaris cells are capable of maintaining the integrity of their cellular membrane, indicating that there is no release of the herbicide from cells. Therefore, $C$. vulgaris could be used in bioremediation systems for freshwater environments polluted with triazine herbicides ( González-Barreiro et al., 2006).

On the basis of the rapid bioaccumulation of herbicide observed in the microalgal biomass, it can be inferred that organisms feeding on pesticide-exposed algae are likely to effectively 
ingest a large proportion of pesticides. In the present study, accumulated levels of terbutryn in the microalgae, even in the lowest concentration assayed, were enough to delay the population growth of the rotifer Brachionus sp. fed with the microalgae. The extent of toxic effects depended on the time of exposure and the accumulated concentration of the toxicant in the microalgae. These results already indicate that, although immersion assays can provide a simple and easily comparable method for assessing toxicity ( Taylor et al., 2005), other factors like the pesticide ingested by food should be taken into account.

Population growth studies provide information on the effect of toxicant on individuals of various generations simultaneously occurring in a growing culture. Population growth rate values can easily be estimated in both laboratory and field settings and can directly be incorporated into ecological models (Preston and Snell, 2001b; Sarma et al., 2005).

In this study, the population growth rate values obtained in the control cultures fall inside the reported range under non-stressful conditions in this species (0.5-2 day-1) (Pavón-Meza et al., 2005), but population growth rates in rotifers fed with terbutryn-exposed microalgae were strongly inhibited when compared to control cultures. Terbutryn at a concentration as low as $12.5 \mathrm{nM}$ had significant effects on population growth rates of the rotifer cultures fed with microalgae exposed to this herbicide. Other studies have determined the effects of heavy metal such as copper (Moreno-Garrido et al., 1999) or hydrocarbons (Wolfe et al., 2001) in a trophic chain similar to this, but there is no current literature that describes the effects of herbicides on rotifer cultures only via food. For example, Sarma et al., 2001a and Sarma et al., 2001breported that the herbicide 2,4-dichlorophenoxyacetic acid had a negative influence on the population growth of $B$. patulus when the rotifers were directly exposed via water and food.

Traditionally, the rate of population increase has received much attention from ecotoxicologists because it is generally more sensitive than short-term and surrogate variables such as mortality (Mangas-Ramirez et al., 2002). However, survival results obtained in this study showed a similar response pattern to the one observed in the population growth rates. The survival curves for all Brachionus sp. cultures fed with terbutryn-exposed microalgae showed a drastic mortality at all concentrations assayed. Moreover, results obtained show that this rotifer species did not survive beyond four days when fed with microalgae exposed to the highest terbutryn concentration assayed, even after being returned to a toxicant-free medium. In general, algae are considered more resistant to toxicants than zooplankton( Kerrison et al., 1988). In fact, the highest concentration of herbicide used here is not toxic to C. vulgaris viability, at least after 24 h of exposure (González-Barreiro et al., 2006), but provokes serious mortality in the rotifer population exposed to it via diet. Terbutryn, which is a triazine herbicide, acts by blocking electron transport in the photosystem II reaction center of the photosynthetic electron transport chain, resulting in the inhibition of photosynthesis. The way in which terbutryn exerts its toxicity to rotifers is not clear. But other terbutryn studies reported alterations to cellular structures other than plant chloroplasts. Moretti et al. (2002) reported genotoxic (primary DNA) damage to human peripheral blood leukocytes following exposure to this herbicide. However, in the rotifer cultures fed with microalgae, which were exposed to 12.5 and $100 \mathrm{nM}$ concentrations, once the 
phase of initial mortality passed, many test individuals reproduced, possibly after some kind of repair to the damaged organs. For that reason, information on the patterns of recovery of zooplankton exposed to different concentrations of toxicants is necessary, but has been little studied. Only similar results were reported with cladocerans that after being exposed to toxicants for a short duration may later recuperate under favourable conditions ( MangasRamirez et al., 2002).

Previous studies of toxicant effects in rotifers have demonstrated that small reductions in population growth rates are caused by reproductive toxicity, that is, by changes in egg ratio (Snell and Serra, 2000). In the present study, at any concentration of terbutryn, the test rotifers failed to reproduce at the same rate as controls, thus, there was a decline in the egg ratio. Egg ratio method is the most distinct indicator of a response of a population to changing ecological conditions and it can thus be used as indicator of stress in ecotoxicological studies on rotifers (Sarma et al., 2005). Depending on the intensity of the toxicant stress, reproduction in rotifer populations can be inhibited with or without affecting the survival of the individuals in the population (Snell and Janssen, 1995). In the present study, under all the tested levels of terbutryn, both reproduction and survival of rotifer populations were adversely affected. When resources become limited, population growth reaches a stationary phase and parthenogenetic egg production declines (Sarma et al., 2005). Egg ratio for a population is usually derived from the total number of parthenogenetic eggs and the total number of females in this population (Edmondson, 1960). Based on our results, during the exponential phase of growth, rotifers have a higher egg production rate per female. Under culture conditions, egg ratio can range from nearly zero, as during the declining phase of the population, to as high as three, if food concentration, temperature and population density conditions are optimal, as was the case in control cultures (Sarma, 1987).

Rotifers are cyclic parthenogens, incorporating both asexual and sexual reproduction into their life cycle (Wallace and Snell, 1991). Given appropriate environmental conditions (temperature, food availability and quality, etc.), asexual females produce sexual females. Sexual females subsequently produce eggs meiotically that develop into haploid males, or resting eggs if fertilized by males (Preston and Snell, 2001a). Although rotifer populations may reach substantial size through asexual reproduction alone, resting egg production through sexual reproduction is essential if rotifers have to survive from one year to the next (Snell and Serra, 2000). Sexual reproduction is density-dependent and, therefore, when environmental conditions are favourable, rapid population growth to a density threshold is necessary for resting egg production. The peak population density reached by a population is an important variable sensitive to toxicant stress (Luna-Andrade et al., 2002). Under terbutryn stress, the peak population densities of Brachionus sp. fed with herbicide pre-accumulating microalgae were significantly lower than those in the controls. This population growth inhibition by pesticide exposure can reduce the probability of resting egg production before the environment deteriorates ( Preston and Snell, 2001b). Males of Brachionus sp. were not observed during this study, indicating absence of sexual reproduction. 
The rotifers populations fed with herbicide-exposed microalgae presented inferior ingestion rates than those observed in the control cultures. The feeding behaviour influences the bioaccumulation of lipophilic compounds significantly (Richter and Nagel, 2007). Since it is closely related to the health of the rotifer populations, a detrimental effect of the herbicide in the rotifer physiology can be assumed. A number of studies have shown that the population density for most rotifer species is directly related to the available food and its quality. For that reason, in this experiment the microalgae remain always at non-limiting levels in all the rotifers cultures. As for food quality, other investigations reported detrimental effects on the population growth of $B$. calyciflorus and $B$. patulus fed with dead Chlorella with respect to the rotifers population density values obtained with live Chlorella ( Pavón-Meza et al., 2001). Based on the results of Chlorella viability described above and unpublished data, obtained in our laboratory, that showed non variations in the biochemical profile of this microalga after $24 \mathrm{~h}$ of exposure to the terbutryn concentrations used in this study, we can conclude that the reduced growth of the rotifer populations fed with terbutryn-exposed microalgae is not related to a loss of the viability or nutritious value of $C$. vulgaris cells.

\section{Conclusion}

This study demonstrates that pollution can result in accumulation of the pollutants by microorganisms, which will in turn alter the normal development of grazer population. However, further studies are necessary because bioaccumulation of pesticides varies with the species of organisms tested based on several factors such as biochemical profile, time of exposure, or surface area for microalgae and, additionally, metabolic capacity and grazing rates for consumer organisms.

\section{Acknowledgements}

This work was carried out with the financial support of the Consellería de Innovación, Industria e Comercio, Xunta de Galicia (PGIDIT04RFO103946PR) and Ministerio de Educación y Ciencia (CGL 2004/02037 BOS). R.P. acknowledges a F.P.U. fellowship from the Spanish Ministerio de Educación y Ciencia.

\section{References}

M.I. Arufe, J. Arellano, M.J. Moreno, C. Sarasquete

Toxicity of a commercial herbicide containing terbutryn and trisulfuron to seabream (Sparus aurata L.) larvae: a comparison with the Microtox test

Ecotoxicol. Environ. Saf., 59 (2004), pp. 209-216

T.E. Brown, F.L. Richardson, M.L. Vaughn

Development of red pigmentation in Chlorococcum wimmeri (Chlorophyta: Chlorococcales)

Phycologia, 6 (1967), pp. 167-184 
R. Carabias-Martínez, E. Rodríguez-Gonzalo, M.E. Fernández-Laespada, L. Calvo-Seronero, F.J. Sánchez-San Román

Evolution over time of the agricultural pollution of waters in an area of Salamanca and Zamora (Spain)

Water Res., 37 (2003), pp. 928-938

\section{W.T. Edmondson}

Reproductive rates of rotifers in natural populations

Memorie dell'Istituto Italiano di Idrobiologia, 12 (1960), pp. 21-77

O. González-Barreiro, C. Rioboo, C. Herrero, A. Cid

Removal of triazine herbicides from freshwater systems using photosynthetic microorganisms

Environ. Pollut., 144 (2006), pp. 266-271

M.Y. Gross-Sorokin, E.P.M. Grist, M. Cooke, M. Crane

Uptake and depuration of 4-Nonylphenol by the benthic invertebrate Gammarus pulex: How important is feeding rate?

Environ. Sci. Technol., 37 (2003), pp. 2236-2241

H. Katsumata, S. Kaneco, T. Suzuki, K. Ohta

Determination of atrazine and simazine in water samples by high-performance liquid chromatography after preconcentration with heat-treated diatomaceous earth

Anal. Chim. Acta, 577 (2006), pp. 214-219

P.H. Kerrison, D. Annoni, S. Zarini, O. Ravera, B. Moss

Effects of low concentration of heavy metals on plankton community dynamics in a small, shallow, fertile lake

J. Plankton Res., 10 (1988), pp. 779-812

Krebs, C.J., 1985. Ecology: the experimental analysis of distribution and abundance, New York, 800 pp. Luna-Andrade et al., 2002

A. Luna-Andrade, R. Aguilar-Duran, S. Nandini, S.S.S. Sarma

Combined effects of copper and microalgal (Tetraselmis suecica) concentrations on the population growth of Brachionus plicatilis Mueller (Rotifera)

Water, Air, Soil Pollut., 141 (2002), pp. 143-153

E. Mangas-Ramirez, S.S.S. Sarma, S. Nandini

Combined effects of algal (Chlorella vulgaris) density and ammonia concentration on the population dynamics of Ceriodaphnia dubia and Moina macrocopa (Cladocera)

Ecotoxicol. Environ. Saf., 51 (2002), pp. 216-222

H.S. Marcial, A. Hagiwara, T.W. Snell

Effect of some pesticides on reproduction of rotifer Brachionus plicatilis Müller Hydrobiologia, 546 (2005), pp. 569-575 


\section{C.P. McCahon, D. Pascoe}

Episodic pollution: causes, toxicological effects and ecological significance

Funct. Ecol., 4 (1990), pp. 375-383

\section{Moreno-Garrido, L.M. Lubián, A.M.V.M. Soares}

In vitro populations of rotifer Brachionus plicatilis Müller demonstrate inhibition when fed with copperpreaccumulating microalgae

Ecotoxicol. Environ. Saf., 44 (1999), pp. 220-225

M. Moretti, M. Marcarelli, M. Villarini, C. Fatigoni, G. Scassellati-Sforzolini, R. Pasquini

In vitro testing for genotoxicity of the herbicide terbutryn: cytogenetic and primary DNA damage

Toxicol. in Vitro, 16 (2002), pp. 81-88

\section{M.G. Ormerod}

Analysis of DNA. General methods

M.G. Ormerod (Ed.), Flow Cytometry. A Practical Approach, Oxford University Press, Oxford (1990), pp. $69-87$

\section{E.L. Pavón-Meza, S.S.S. Sarma, S. Nandini}

Effect of different densities of live and dead Chlorella vulgaris on the population growth of rotifers Brachionus calyciflorus and Brachionus patulus (Rotifera)

Rev. Biol. Trop., 49 (2001) 895-602

\section{E.L. Pavón-Meza, S.S.S. Sarma, S. Nandini}

Combined effects of different densities of live and dead Chlorella vulgaris on the population growth of rotifers Brachionus calyciflorus and Brachionus patulus (Rotifera)

Rev. Biol. Trop., 49 (2005) 895-602

\section{B.L. Preston, T.W. Snell}

Full life-cycle toxicity assessment using rotifer resting egg production: implications for ecological risk assessment

Environ. Pollut., 114 (2001), pp. 399-406

B.L. Preston, T.W. Snell

Direct and indirect effects of sublethal toxicant exposure on population dynamics of freshwater rotifers: a modeling approach

Aquat. Toxicol., 52 (2001), pp. 87-99

\section{S. Richter, R. Nagel}

Bioconcentration, biomagnification and metabolism of 14C-terbutryn and 14C-benzo[a]pyrene in Gammarus fossarum and Aselles aquaticus

Chemosphere, 66 (2007), pp. 603-610

\section{S.S.S. Sarma}


Experimental Studies On the Ecology of Brachionus patulus (Müller) (Rotifera) in Relation to Food, Temperature and Predation

University of Delhi, Delhi, India (1987)

\section{S.S.S. Sarma, S. Nandini, J.L. Gama Flores}

Effect of methyl parathion on the population growth of the rotifer Brachionus patulus (O. F. Müller) under different algal food (Chlorella vulgaris)

Ecotoxicol. Environ. Saf., 48 (2001), pp. 190-195

S.S.S. Sarma, R.D. Gulati, S. Nandini

Factors affecting egg-ratio in planktonic rotifers

Hydrobiologia., 546 (2005), pp. 361-373

S.S.S. Sarma, T. Ramirez-Perez, S. Nandini, I. Penalosa-Castro

Combined effects of food concentration and the herbicide 2,4-dichlorophenoxyacetic acid on the population dynamics of Brachionus patulus (Rotifera)

Ecotoxicology, 10 (2001), pp. 91-99

T.W. Snell, C.R. Janssen

Rotifers in ecotoxicology: a review

Hydrobiologia, 313-314 (1995), pp. 231-247

T.W. Snell, M. Serra

Using probability of extinction to evaluate the ecological significance of toxicant effects

Environ. Toxicol. Chem., 19 (2000), pp. 2357-2363

J. Tang, K.D. Hoagland, B.D. Siegfried

Uptake and bioconcentration of atrazine by selected freshwater algae

Environ. Toxicol. Chem., 17 (1998), pp. 1085-1090

R.L. Taylor, G.S. Caldwell, M.G. Bentley

Toxicity of algal-derived aldehydes to two invertebrate species: Do heavy metal pollutants have a synergistic effect?

Aquat. Toxicol., 74 (2005), pp. 20-31

C. Tomlin

The Pesticide Manual. A World Compendium

BCPC (Brithish Crop Protection Council), Hampshire, UK (2003) 1341 pp

R.L. Wallace, T.W. Snell

Rotifera.

J.H. Thorp, A.P. Covich (Eds.), Ecology and Systematics of North American Freshwater Invertebrates, Academic Press, New York, USA (1991), pp. 187-248 
M.F. Wolfe, G.J.B. Schwartz, S. Singaram, E.E. Mielbrecht, R.S. Tjeerdema, M.L. Sowby

Influence of dispersants on the bioavailability and trophic transfer of petroleum hydrocarbons to larval topsmelt (Atherinops affinis)

Aquat. Toxicol., 52 (2001), pp. 49-60

Corresponding author. Tel.: +34 981 167000; fax: +34 981167065 .

Copyright () 2007 Elsevier B.V. All rights reserved. 